\title{
KL-MOB: automated COVID-19 recognition using a novel approach based on image enhancement and a modified MobileNet CNN
}

\author{
Mundher Mohammed Taresh ${ }^{1}$, Ningbo Zhu ${ }^{\text {Corresp., }}{ }^{1}$, Talal Ahmed Ali Ali ${ }^{1}$, Mohammed Alghaili ${ }^{1}$, Asaad Shakir \\ Hameed $^{2}$, Modhi Lafta Mutar $^{2}$ \\ ${ }^{1}$ College of Information Science and Engineering, Hunan University, Changsha, Hunan, China \\ 2 Department of Mathematics, General Directorate of Thi-Qar Education, Ministry of education, Thi-Qar, Iraq \\ Corresponding Author: Ningbo Zhu \\ Email address: quietwave@hnu.edu.cn
}

The emergence of the novel coronavirus pneumonia (Covid-19) pandemic at the end of 2019 led toworldwide chaos. However, the world breathed a sigh of relief when a few countries announced the development of a vaccine and gradually began to distribute it. Nevertheless, the emergence of another wave of this pandemic returned us to the starting point. At present, early detection of infected people is the paramount concern of both specialists and health researchers. This paper proposes a method to detect infected patients through chest $\mathrm{x}$-ray images by using the large dataset available online for Covid-19 (COVIDx), which consists of 2128 X-ray images of Covid-19 cases, 8066 normal cases, and 5575 cases of pneumonia. A hybrid algorithm is applied to improve image quality before undertaking neural network training. This algorithm combines two different noise-reduction filters in the image, followed by a contrast enhancement algorithm. To detect Covid-19, we propose a novel convolution neural network (CNN) architecture called KL-MOB (Covid-19 detection network based on the MobileNet structure). The performance of $\mathrm{KL}-\mathrm{MOB}$ is boosted by adding the Kullback-Leibler $(\mathrm{KL})$ divergence loss function when trained from scratch. The KL divergence loss function is adopted for content-based image retrieval and fine-grained classification to improve the quality of image representation. The results are impressive: the overall benchmark accuracy, sensitivity, specificity, and precision are $98.7 \%, 98.32 \%, 98.82 \%$, and $98.37 \%$, respectively. These promising results should help other researchers develop innovative methods to aid specialists. The tremendous potential of the method proposed herein can also be used to detect Covid-19 quickly and safely in patients throughout the world. 


\title{
KL-MOB: Automated Covid-19 Recognition Using a Novel Approach Based on Image Enhancement and a Modified MobileNet CNN
}

\author{
Mundher Mohammed Taresh ${ }^{1}$, Ningbo Zhu ${ }^{1}$, Talal Ahmed Ali Ali ${ }^{1}$, \\ Mohammed Alghaili ${ }^{1}$, Asaad Shakir Hameed ${ }^{2}$, and Modhi Lafta Mutar ${ }^{2}$ \\ ${ }^{1}$ College of Information Science and Engineering, Hunan University, Changsha, Hunan, \\ China \\ ${ }^{2}$ Department of Mathematics, General Directorate of Thi-Qar Education, Ministry of \\ education, Thi-Qar, Iraq \\ Corresponding author: \\ Ningbo Zhu ${ }^{1}$ \\ Email address: quietwave@hnu.edu.cn
}

\begin{abstract}
The emergence of the novel coronavirus pneumonia (Covid-19) pandemic at the end of 2019 led to worldwide chaos. However, the world breathed a sigh of relief when a few countries announced the development of a vaccine and gradually began to distribute it. Nevertheless, the emergence of another wave of this pandemic returned us to the starting point. At present, early detection of infected people is the paramount concern of both specialists and health researchers. This paper proposes a method to detect infected patients through chest $x$-ray images by using the large dataset available online for Covid19 (COVIDx), which consists of 2128 x-ray images of Covid- 19 cases, 8066 normal cases, and 5575 cases of pneumonia. A hybrid algorithm is applied to improve image quality before undertaking neural network training. This algorithm combines two different noise-reduction filters in the image, followed by a contrast enhancement algorithm. To detect Covid-19, we propose a novel convolution neural network (CNN) architecture called KL-MOB (Covid-19 detection network based on the MobileNet structure). The performance of KL-MOB is boosted by adding the Kullback-Leibler $(\mathrm{KL})$ divergence loss function when trained from scratch. The KL divergence loss function is adopted for content-based image retrieval and fine-grained classification to improve the quality of image representation. The results are impressive: the overall benchmark accuracy, sensitivity, specificity, and precision are $98.7 \%, 98.32 \%, 98.82 \%$, and $98.37 \%$, respectively. These promising results should help other researchers develop innovative methods to aid specialists. The tremendous potential of the method proposed herein can also be used to detect Covid-19 quickly and safely in patients throughout the world.
\end{abstract}

\section{INTRODUCTION}

The novel coronavirus 2019 (Covid-19) is a recently recognized disease caused by the severe acute respiratory syndrome coronavirus 2 (SARS-CoV-2). Being highly transmissible and life-threatening, it has rapidly turned into a global pandemic, affecting worldwide health and well-being. Tragically, no effective treatment has yet been approved for patients with Covid-19. However, patients can have a good chance of survival if they are diagnosed sufficiently early, where they would undergo the plan of remedial measures correctly.

As a widely available, time- and cost-effective diagnostic tool, chest X-rays (CXRs) can potentially be used for early recognition of Covid-19. Nevertheless, Covid-19 can share similar radiographic features with other types of pneumonia, making it difficult for radiologists to manually distinguish between the two. As a result, manual detection of Covid-19 is time-consuming and mistake-prone because it is left to the subjective judgment of the radiologist. It is thus highly desirable to develop automated detection 
techniques.

With the rapid global spread of Covid-19, researchers have begun using state-of-the-art deep-learning techniques to automate the recognition of Covid-19. The initial lack of Covid-19 data compelled earlier researchers to use pretrained networks to build their own models (Narin et al., 2020; Ozturk et al., 2020; Apostolopoulos and Mpesiana, 2020; Civit-Masot et al., 2020; Albahli, 2020; Sethy and Behera, 2020; Apostolopoulos et al., 2020; Chowdhury et al., 2020; Farooq and Hafeez, 2020; Maghdid et al., 2020; Hemdan et al., 2020; Taresh et al., 2021; Punn and Agarwal, 2021). Given that Covid-19 infected millions of people worldwide within a few months of its detection, a mid-range dataset of positive cases was made available for public use (Wang et al., 2020). This dataset can be uploaded from https://github. com/lindawangg/COVID-Net/blob/master/docs/COVIDx.md. This, in turn, has enabled further progress in developing new, accurate, in-depth models for Covid-19 recognition (Ahmed et al., 2020; Afshar et al., 2020; Ucar and Korkmaz, 2020; Luz et al., 2020; Hirano et al., 2020; Rezaul Karim et al., 2020). However, some medical imaging issues usually pose difficulties in the recognition task, reducing the performance of these models. These issues include, but are not limited to, insufficient training data, inter-class ambiguity, intra-class variation, and visible noise. These problems oblige us to significantly enhance the discrimination capability of the associated model. Specifically, regarding the $\mathrm{x}$-ray image, the common characteristics are grayscale color space, high noise, low intensity, poor contrast, and weak boundary representation, which will normally affect the information of the image (Ikhsan et al., 2014).

One way around these issues is to use proper image preprocessing techniques for noise reduction and contrast enhancement. A closer look at the available images reveals the presence of various types of noise, such as impulsive, Poisson, speckle, and Gaussian noise [see Figure 1 for the most common types of noise in x-ray images (Paul et al., 2018)]. However, the most prevalent studies have focused only on some of these types of noise (e.g., Gaussian and Poisson). In particular, among many other techniques, histogram equalization (HE) (Civit-Masot et al., 2020; Tartaglione et al., 2020; Rezaul Karim et al., 2020), contrast limited adaptive histogram equalization (CLAHE) (El-bana et al., 2020; Saiz and Barandiaran, 2020; Maguolo and Nanni, 2021; Ramadhan et al., 2020), adaptive total variation method(ATV) (Punn and Agarwal, 2021), white balance followed by CLAHE (Siddhartha and Santra, 2020), intensity normalization followed by CLAHE (N-CLAHE) (Horry et al., 2020; El Asnaoui and Chawki, 2020), Perona-Malik filter (PMF), unsharp masking (UM) (Rezaul Karim et al., 2020), Bihistogram equalization with adaptive sigmoid function (BEASF) (Haghanifar et al., 2020), the gamma correction (GC) (Rahman et al., 2021), histogram stretching (HS) (Wang et al., 2021; Zhang et al., 2021), Moment Exchange algorithm (MoEx), CLAHE (Lv et al., 2021), local phase enhancement (LPE) (Qi et al., 2021), image contrast enhancement algorithm (ICEA) (Canayaz, 2021), and Gaussian filter (Medhi et al., 2020) are, as far as we are aware, the only adopted techniques in Covid-19 recognition to date. An overview of these works is listed in Table 1. It should be noted that the CLAHE algorithm has widely used by the majority, while some pursued a hybridization method. Moreover, the utilized filters can result in blurry (by Gaussian filter) or blocky (by PMF) features in the processed image. Accordingly, there is still room to incorporate more effective preprocessing techniques to further increase the accuracy of these systems.

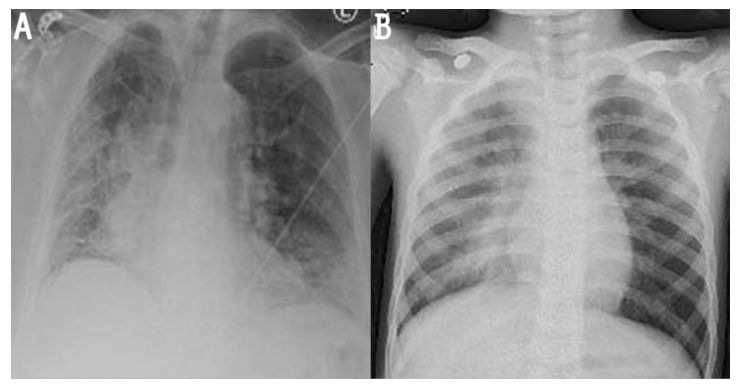

Figure 1. Noisy images: (A) image with impulsive noise and (B) image with Gaussian noise.

Motivated by the outstanding results in the previously mentioned works as well as the need for closeto-perfect recognition models, this paper integrates novel image preprocessing enhancement with deep learning to meet the challenges arising from data deficiency and complexity. Specifically, we combine 


\begin{tabular}{c|c|c} 
Study & Image enhancement appraoch & Method \\
\hline Civit-Masot et al. (2020) & HE & VGG16 \\
Tartaglione et al. (2020) & HE & ResNet18, ResNet50, DenseNet121 \\
Ramadhan et al. (2020) & CLAHE & COVIDLite \\
El-bana et al. (2020) & CLAHE & VGG16 \\
Saiz and Barandiaran (2020) & CLAHE & AlexNet \\
Maguolo and Nanni (2021) & CLAHE & ResNet, InceptionV3, InceptionResNetV2, \\
Punn and Agarwal (2021) & ATV & DenseNet169, and NASNetLarge \\
Siddhartha and Santra (2020) & White balance, CLAHE & VGIDLite \\
Horry et al. (2020) & N-CLAHE & VGG19 \\
El Asnaoui and Chawki (2020) & CLAHE & VGG16, VGG19, DenseNet201, InceptionResNetV2, \\
Rezaul Karim et al. (2020) & HE, PMF, UM & InceptionV3, Resnet50, and MobileNetV2 \\
Medhi et al. (2020) & Gaussian filtering & DeepCOVIDExplainer \\
Haghanifar et al. (2020) & CLAHE, BEASF & Deep CNN \\
Rahman et al. (2021) & GC & COVID-CXNet (UNet+DenseNet) \\
Wang et al. (2021) & HS & Seven different deep CNN networks for classification \\
Zhang et al. (2021) & HS & andified Unet network for segmentation \\
Lv et al. (2021) & PoEx, CLAHE & Deep convolutional attention network \\
Qi et al. (2021) & LPE & Cascade-SEME net \\
Canayaz (2021) & ICEA & Fus-ResNet50 \\
\end{tabular}

Table 1. An overview of image enhancement techniques and the deep learning method used for Covid-19 detection.

an adaptive median filter (AMF) and a non-local means filter (NLMF) to remove the noise from the images. Numerous works have already analyzed the performance of these two filters for denoising x-ray imagery (Kim et al., 2020; Raj and Venkateswarlu, 2012; Rabbouch et al., 2020; Sawant et al., 1999; Mirzabagheri, 2017), demonstrating their superiority over various filters, including the ones in the cited works in terms of removing impulsive, Poisson, and speckle noise while preserving the useful image details. We then utilize the CLAHE approach that has been already applied for the enhancement of contrast in medical images (Zhou et al., 2016; Sonali et al., 2019; Wen et al., 2016), to enhance the contrast of the denoised images. The enhanced images are finally fed into the state-of-the-art convolution neural network (CNN) called MobileNet (Howard et al., 2017), which has been recently utilized for the same classification task by (Apostolopoulos et al., 2020; Apostolopoulos and Mpesiana, 2020). MobileNets are small, low-latency, low-power models parameterized to meet the resource constraints of a variety of use cases. The motivation behind choosing a MobileNet CNN is that it not only helps to reduce overfitting but also runs faster than a regular CNN and has significantly fewer parameters (4.24) (Howard et al., 2017; Yu et al., 2020). Moreover, MobileNets employ two global hyperparameters based on depthwise separable convolutions to strike a balance between efficiency and accuracy.

KL divergence is one of the measures that reflect the distribution divergence between different probabilities, which has been widely used in the problem of classification imbalanced datasets ( $\mathrm{Su}$ et al., 2015; Feng et al., 2018). The KL divergence loss function is more commonly used when using models that learn to approximate a more complex function than simply multiclass classification, such as in the case of an autoencoder used for learning a dense feature representation under a model that must reconstruct the original input. Indeed, the lack of necessary extracted features from the images sometimes cannot provide expected accuracy in the classification result. In this work, inspired by the variational autoencoder learning (Kingma and Welling, 2013; Alfasly et al., 2019; Alghaili et al., 2020) the Kullback-Leibler (KL) divergence is adopted to devise more efficient and accurate representations and measure how far we are from the optimal solution during the iterations. We evaluated the performance of the proposed framework on the COVIDx dataset in terms of a wide variety of metrics: accuracy, sensitivity, specificity, precision, area under the curve, and computational efficiency. Simulation results reveal that the proposed framework significantly outperforms state-of-the-art models from both quantitative and qualitative perspectives.

The novelty of this study is not only to clarify significant features in the CXR images by developing a hybrid algorithm but also proposes a novel approach in how to devise more efficient and accurate by using KL loss. The intent behind this study is not only to achieve a high classification accuracy but to 
achieve this by training an automated end-to-end deep learning framework based on CNN. This method is superior to transfer learning for evaluating the importance of features derived from imagery, as it is not relying on features previously learned by the pretrained model, which was first trained on nonmedical images. The main contributions of this work can be summarized as follows:

- For Covid-19 recognition, we propose an automated end-to-end deep learning framework based on MobileNet CNN with KL divergence loss function.

- We propose an impressive approach to ensure a sufficiently diverse representation by predicting the output of the mean $\mu$ and standard-deviation $\sigma$ of the Gaussian distribution.

- We incorporate a novel preprocessing enhancement technique consisting of AMF, NLMF, and CLAHE to meet the challenges arising from data deficiency and complexity.

- We analyze the performance of the preprocessing enhancement scheme to demonstrate its role in enhancing the discrimination capability of the proposed model.

The rest of this paper is organized as follows: Section (2) describes the phases of the proposed method. Section (3) highlights the experimental results. Section (4) discusses these results, and the conclusion is presented in Section (5).

\section{PROPOSED METHOD}

In this section, we briefly describe the scenario of the methodology used to achieve the purpose of this study. The proposed method is depicted in Figure 2, which generally consists of two phases: (a) image preprocessing, to overcome the existing drawbacks mentioned in the previous section; (b) training and testing dedicated to image classification.

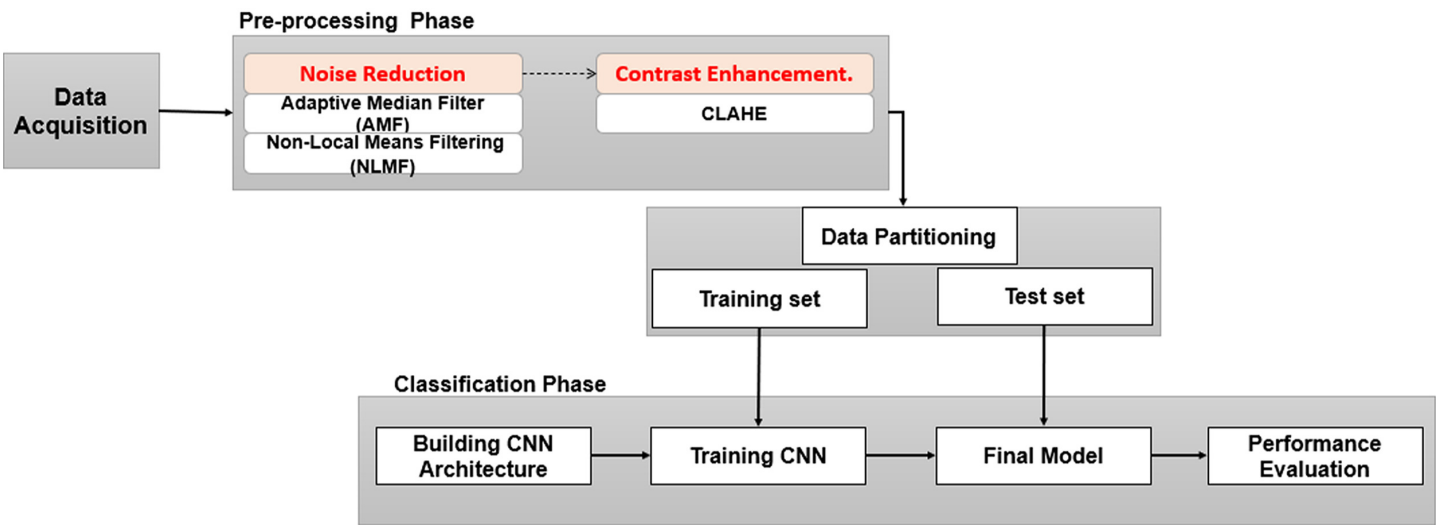

Figure 2. Framework of study.

\section{Data Acquisition}

In this work, we used the COVIDx dataset used by (Wang et al., 2020) to train and evaluate the proposed model. In brief, the COVIDx dataset is an open-source dataset that can be downloaded from https : //github.com/lindawangg/COVID-Net/blob/master/docs/COVIDx. md. The instructions given by (Wang et al., 2020) were followed to set up the new dataset. Since few CXR images of positive Covid-19 cases are available, we downloaded more Covid-19 x-ray images from https : / / g i thub . $\mathrm{com} / \mathrm{ml}$-workgroup/covid-19-image-repository, and from https://github.com/ armiro/COVID-CXNet/tree/master/chest_xray_images/covid19. Duplicated images were omitted from the new dataset to ensure that the proposed training model is more accurate. Thus, the actual number of images in the Covid-19 class is 2128 instead of the 1770 images from COVIDx (updated on January 28, 2021). We used the same test set that was used for evaluation by (Wang et al., 2020), making only a slight change by increasing the number of Covid-19 images to 100 instead of 92 .We further split the training data keeping $70 \%$ data for training and 30\% data for validation. Table 2 summarizes the number of images in each class and the total number of images used for training and testing. 


\begin{tabular}{c|c|c|c|c} 
Classes & Total & $\begin{array}{c}\text { Training set } \\
70 \%\end{array}$ & $\begin{array}{c}\text { Validation set } \\
30 \%\end{array}$ & $\begin{array}{c}\text { Test set } \\
\text { (unseen) }\end{array}$ \\
\hline Covid-19 & 2128 & 1420 & 608 & 100 \\
Normal & 8066 & 5027 & 2154 & 885 \\
Pneumonia & 5575 & 3487 & 1494 & 594 \\
Total & 15769 & 9933 & 4257 & 1579
\end{tabular}

Table 2. The number of images for each class.

\section{Data Preprocessing Method}

In this study, we attempt to provide an algorithm that would increase the image quality by using a hybrid technique consisting of noise reduction and contrast enhancement. Specifically, two efficient filters are used for noise reduction while CLAHE is used for contrast enhancement. The first filter is the AMF, which removes impulse noise (Ning et al., 2009; Khare and Chugh, 2014). This filter is followed by the NLMF algorithm that calculates similarity based on patches instead of pixels. Given a discrete noisy image $u=u(i)$ for pixel $I$, the estimated value of $N L[u](i)$ is the weighted average of all pixels:

$$
N L[u](i)=\sum_{j \in i} w(i, j) \cdot u(j)
$$

where the weight family $w(i, j) j$ depends on the similarity between the pixels $i$ and $j$.

The similarity between the two pixels $i$ and $j$ is defined by the similarity of the intensity of gray-level vectors $u\left(N_{i}\right)$ and $u\left(N_{j}\right)$, where $N_{l}$ signifies a square neighborhood of fixed size and centered at a pixel $L$. The similarity is measured as a function to minimize the weighted Euclidean distance, $\left\|u\left(N_{i}\right)-u\left(N_{j}\right)\right\|_{(2, a)}^{2}$ where $a>0$ is the Gaussian kernel standard deviation. The pixels with a similar gray-level neighborhood with $u\left(N_{i}\right)$ have larger weights in average. These weights are defined as;

$$
w(i, j)=\frac{1}{Z(i)} e^{-\frac{\left\|u\left(N_{i}\right)-u\left(N_{j}\right)\right\|_{(2, a)}^{2}}{h^{2}}}
$$

where $Z(i)$ is the normalizing constant: $Z(i)=\sum_{j} e^{-\frac{\left\|u\left(N_{i}\right)-u\left(N_{j}\right)\right\|_{(2, a)}^{2}}{h^{2}}}$, and the parameter $h$ acts as a degree of filtering.

Next, CLAHE is applied to the denoised images to achieve an acceptable visualization and to compensate for the effect of filtration that may contribute to some blurring on the images (Huang et al., 2016; Senthilkumar and Senthilmurugan, 2014). Since there are many homogeneous regions in medical images, CLAHE is suitable for optimizing medical images as the CLAHE algorithm creates non-overlapping homogeneous regions.

\section{Classification Neural Network Model}

We used a deep neural network structure called a MobileNet neural network (Howard et al., 2017). All images were resized to $224 \times 224 \times 3$ before being used as input to the neural network. Figure 3 depicts the architecture of the proposed neural network. 


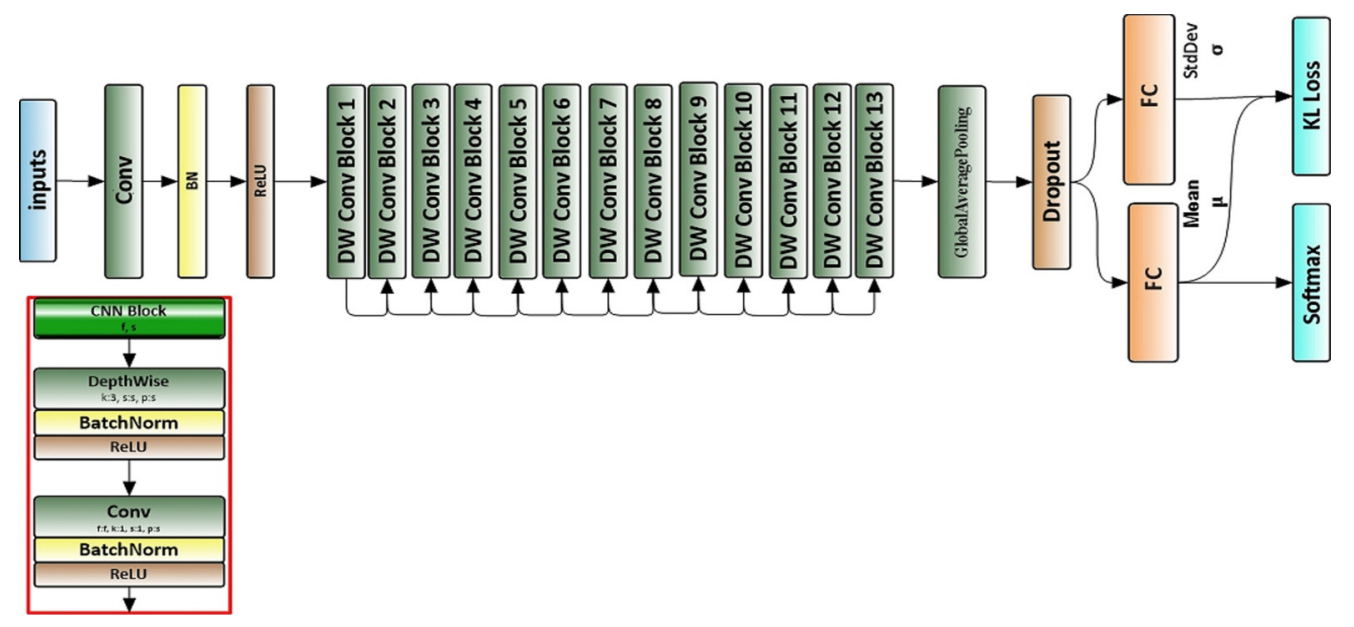

Figure 3. Architecture of proposed neural network.

Apart from the first layer, which is a full convolution, the MobileNets are constructed using depthwise separable convolutions. Depthwise separable convolution is a factorized convolution that factorizes the standard convolution into a depthwise convolution and a $1 \times 1$ convolution called pointwise convolution. This procedure reduces the computations and model size drastically. The overall architecture of the MobileNet is shown in Table 3.

\begin{tabular}{c|c|c|c|c} 
Type & Stride & Filter Shape & Size in & Size out \\
\hline Conv1 & 2 & $3 \times 3 \times 3 \times 32$ & $224 \times 224 \times 3$ & $112 \times 112 \times 32$ \\
Conv2 dw & 1 & $3 \times 3 \times 32$ & $112 \times 112 \times 32$ & $112 \times 112 \times 32$ \\
Conv2 pw & 1 & $1 \times 1 \times 32 \times 64$ & $112 \times 112 \times 32$ & $112 \times 112 \times 64$ \\
Conv3 dw & 2 & $3 \times 3 \times 64$ & $112 \times 112 \times 64$ & $56 \times 56 \times 64$ \\
Conv3 pw & 1 & $1 \times 1 \times 64 \times 128$ & $56 \times 56 \times 64$ & $56 \times 56 \times 128$ \\
Conv4 dw & 1 & $3 \times 3 \times 128$ & $56 \times 56 \times 128$ & $56 \times 56 \times 128$ \\
Conv4 pw & 1 & $1 \times 1 \times 128 \times 128$ & $56 \times 56 \times 128$ & $56 \times 56 \times 128$ \\
Conv5 dw & 2 & $3 \times 3 \times 128$ & $56 \times 56 \times 128$ & $56 \times 56 \times 128$ \\
Conv5 pw & 1 & $1 \times 1 \times 128 \times 256$ & $28 \times 28 \times 128$ & $28 \times 28 \times 128$ \\
Conv6 dw & 1 & $3 \times 3 \times 256$ & $28 \times 28 \times 256$ & $28 \times 28 \times 265$ \\
Conv6 pw & 1 & $1 \times 1 \times 256 \times 256$ & $28 \times 28 \times 256$ & $28 \times 28 \times 256$ \\
Conv7 dw & 2 & $3 \times 3 \times 256$ & $28 \times 28 \times 256$ & $14 \times 14 \times 256$ \\
Conv7 pw & 1 & $1 \times 1 \times 256 \times 512$ & $14 \times 14 \times 256$ & $14 \times 14 \times 512$ \\
Conv8-12 dw & 1 & $3 \times 3 \times 512$ & $14 \times 14 \times 512$ & $14 \times 14 \times 512$ \\
Conv8-12 pw & 1 & $1 \times 1 \times 512 \times 512$ & $14 \times 14 \times 512$ & $14 \times 14 \times 512$ \\
Conv13 dw & 2 & $3 \times 3 \times 512$ & $14 \times 14 \times 512$ & $7 \times 7 \times 512$ \\
Conv13 pw & 1 & $1 \times 1 \times 512 \times 1024$ & $7 \times 7 \times 512$ & $7 \times 7 \times 1024$ \\
Conv14 dw & 2 & $3 \times 3 \times 1024$ & $7 \times 7 \times 1024$ & $7 \times 7 \times 1024$ \\
Conv14 pw & 1 & $1 \times 1 \times 1024 \times 1024$ & $7 \times 7 \times 1024$ & $7 \times 7 \times 1024$ \\
GAP & 1 & Pool $7 \times 7$ & $7 \times 7 \times 1024$ & $1 \times 1 \times 1024$ \\
Dropout & 1 & Probability=0.001 & $1 \times 1 \times 1024$ & $1 \times 1 \times 1024$ \\
FC $(\mu)$ & 1 & $128 \times 3$ & $1 \times 1 \times 1024$ & $1 \times 1 \times 128$ \\
FC $(\sigma)$ & 1 & $128 \times 3$ & $1 \times 1 \times 1024$ & $1 \times 1 \times 128$ \\
Softmax & 1 & Classifier & $1 \times 1 \times 128$ & $1 \times 1 \times 3$
\end{tabular}

Table 3. Layers of prposed CNN model architecture.

The deep convolutional neural network is used to extract high context features per input instance. The global average pooling layer is used here to reduce the spatial dimensions of the features extracted. The output is a feature vector of size 1024 for each time step. Then, a dropout layer is used with a probability of 0.001 . The output of the dropout layer goes to two fully connected layers that generate an output of size 128 . One fully connected layer is used to predict the mean $\mu$, which is used to extract the most significant features from those features extracted in previous layers. The other is used to predict the 
standard deviation $\sigma$ of a Gaussian distribution, which is used to calculate the KL loss function. The output of the fully connected layer, which used to predict the mean $\mu$ goes to the last layer (Softmax classifier), which is defined by

$$
L_{C E}(o, v)=-\sum_{i=1}^{v} o_{i} \log \left(\frac{e^{p i}}{\sum_{j}^{v} e^{p j}}\right)
$$

where $v$ indicates the output vector, $o$ indicates the objective vector, and $p j$ indicates the input to the neuron $j$.

The categorical cross-entropy loss function is generally used to address such a multiclass classification problem. The three classes are provided with labels such as "0" being a Covid-19 case, " 1 " being a normal case, and " 2 " being pneumonia. We adopted Kullback-Leibler divergence loss function to devise more efficient and accurate representations. Moreover, the combined KL loss with the categorical cross-entropy loss function would enforce the network to give a consistent output, in addition to the preprocessing applied to the input image. The KL divergence distribution between the $\mu ; \sigma$ and the prior is considered as a regularization that aids in addressing the issue of overfitting. KL loss function is defined by

$$
D_{K L}=-\frac{1}{2} \sum_{i=1}^{n}\left(1+\log \left(\sigma_{i}\right)-\mu_{i}^{2}-\sigma_{i}\right)
$$

where $n$ is the output vector of the average pooling layer with the size of $1024, \mu$ is the mean, which is predicted from one fully connected layer, and $\sigma$ is the standard deviation of a Gaussian distribution, which is predicted from the other fully connected layer in the network, Figure 3. The multitask learning loss function for our proposed network is now defined by

$$
L=\alpha D_{K L}+L_{C E}(o, v)
$$

We use a weighted loss function as illustrated in Equation 5. The weight of KL loss $\alpha$ is empirically set to $(0: 1)$ to be used as a one-hot vector, which not only ensures a clear representation of the true class, but also helps in addressing the large variance arising due to unbalanced data.

\section{Experiments}

All CXRs were resized to the same dimension of $224 \times 224$ in .jpg format. In the first phase, the AMF window size was taken to be $5 \times 5$ for effective filtering. The resultant image was then subjected to the NLMF technique. The performance of the NLMF was depended on $7 \times 7$ of the search window, $5 \times 5$ of the similarity window, and a degree of filtering $h=1$. Furthermore, we increased the contrast using CLAHE with the bin of 256 and block size of 128 in slope 3 to get the enhanced images. We passed the images to KL-MOB as the input to predict the CXR image (Covid-19, normal, or pneumonia). Because many functions are not built-in functions from deep learning libraries, such as the relu6 activation function with a max value of six, we built an interface for the evaluation process that contains all layers in the network, as in a training network, but which is not used for training. Instead, it is used to pass on the input image to produce the output.

The proposed model (KL-MOB) is implemented by using the Python programming language. All experiments were conducted on a Tesla K80 GPU graphics card on Google Collaboratory with an Intel $\odot$ i7-core @3.6GHz processor and 16GB RAM with 64-bit Windows 10 operating system. The original and enhanced images are used separately to train the KL-MOB. In the first stage, the baseline model is trained to verify the influence of the KL loss on performance. Figure 4 presents the curve comparisons of all training processes. With the maximum training epoch set to 200. A large gap between training and validation in both original and enhanced images indicates the presence of overfitting.

The network is trained by using a SoftMax classifier with an Adam optimizer (Kingma and Ba, 2014) with the initial learning rate set to 0.0001 and a batch size of 32 . The dataset used for training is divided into $70 \%$ as a training set and $30 \%$ as a validation set. The total number of parameters is $3,488,426$, where the number of trainable parameters is $3,466,660$, and the nontrainable parameters are 21,766 . In 

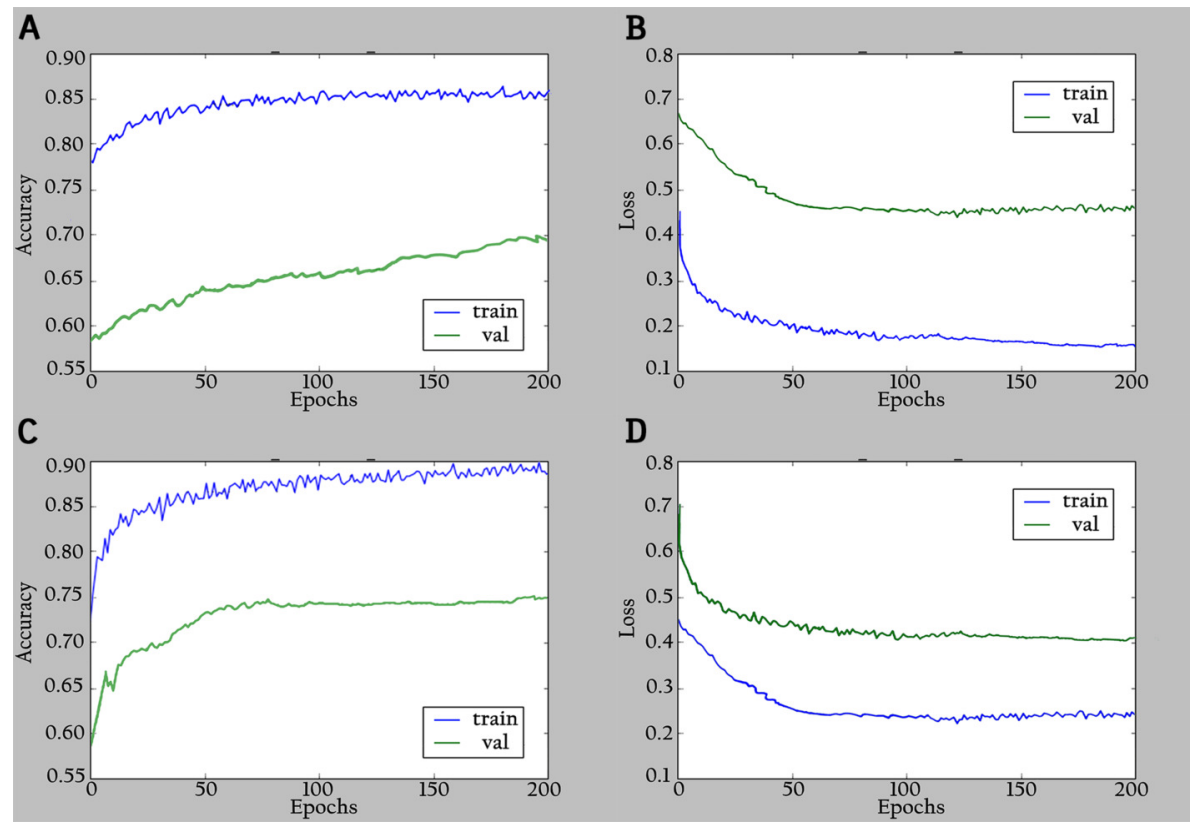

Figure 4. Accuracy and loss graphs for baseline model:(A) training and validation accuracy of the original images, (B) training and validation loss of the original images, (C) training and validation accuracy of the enhanced images and (D) training and validation loss of the enhanced images.

the training period, 200 epochs were completed to check the KL-MOB model accuracy and loss, which are shown in Figures 5 and 6.
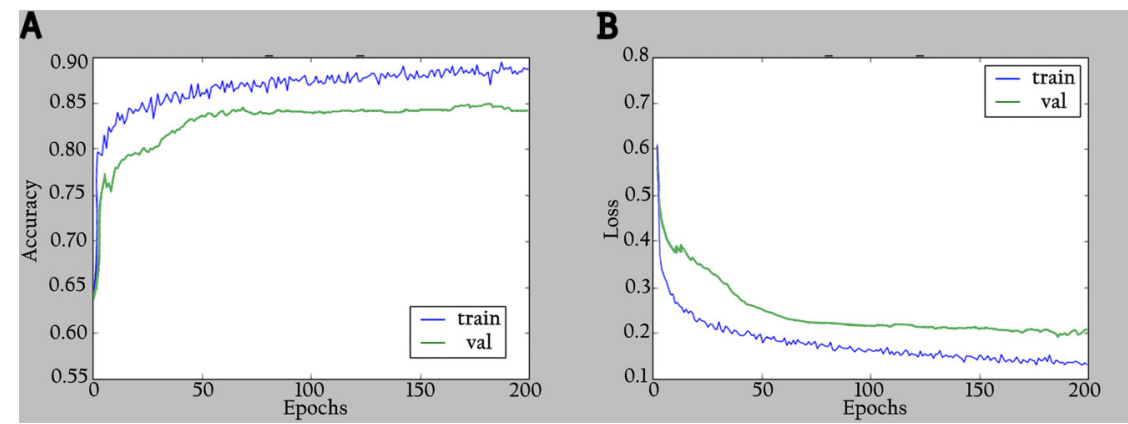

Figure 5. Accuracy and loss graphs for KL-MOB on training and validation of the original images: (A) accuracy and (B) loss.
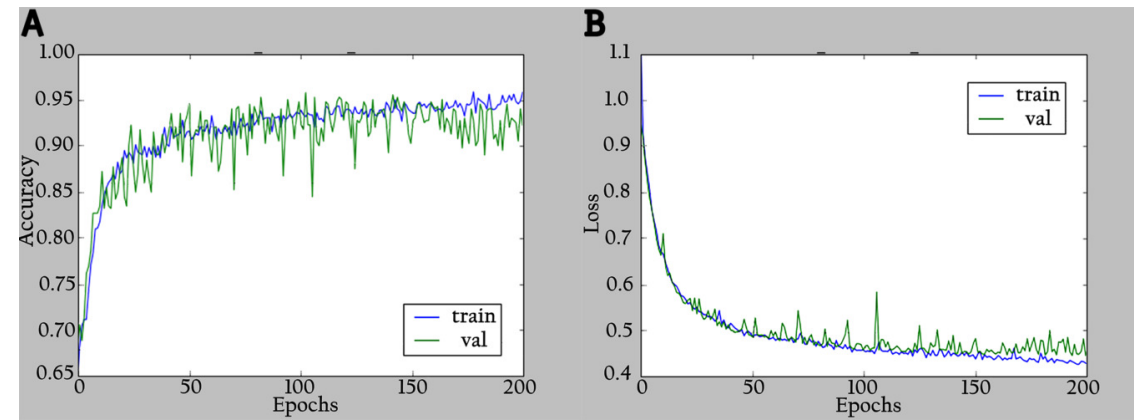

Figure 6. Accuracy and loss graphs for KL-MOB on training and validation of the enhanced images: (A) accuracy and (B) loss. 
Beforehand, the impact of different feature sizes on training accuracy has been investigated via conducting extensive experiments. Original images perform best when the length is set to 256, with an accuracy of $93.24 \%$, whereas the enhanced images perform best when the length is set to 128 with an accuracy of $96.06 \%$, as shown in Table 4 . This can be attributed to the fact that the KL divergence between $\mu ; \sigma$ distribution and the prior is considered as a regularization which helps to overcome the overfitting problem.

\begin{tabular}{c|c|cc|}
\multirow{2}{*}{ Model } & \multirow{2}{*}{ Output vector } & \multicolumn{2}{|c|}{ Accuracy\% } \\
\cline { 3 - 4 } & & enhanced & original \\
\hline \multirow{4}{*}{ KL-MOB } & 64 & 93.26 & 88.31 \\
& 128 & 96.06 & 89.36 \\
& 256 & 95.87 & 93.24 \\
& 512 & 94.83 & 91.08 \\
& 1024 & 94.47 & 90.38
\end{tabular}

Table 4. Model performance on different feature sizes.

\section{Performance Evaluation}

\section{Preprocessing Performance Evaluation}

The performance of the proposed preprocessing technique was quantified by using various evaluation metrics such as mean average error (MAE) and peak signal-to-noise Ratio (PSNR). These metrics are desirable because they can be rapidly quantified.

Definition: $x(i, j)$ denotes the samples of the original image, $y(i, j)$ denotes the samples of the output image. $M$ and $N$ are the number of pixels in row and column directions, respectively. $M A E$ is calculated as in Equation 6, where a large value means that the images are of poor quality.

$$
M A E=|E(x)-E(y)|,
$$

The limited value PSNR implies that the images are of low quality. PSNR is described in terms of Mean Square Error MSE as follows:

$$
P S N R=10 \log _{10} \frac{M A X^{2}}{M S E}
$$

where $M A X^{2}$ is the maximum possible pixel intensity value 255 when the pixel is represented by 8 bits.

$$
M S E=\sqrt{\frac{1}{M N} \sum_{i=1}^{M-1} \sum_{j=1}^{N-1}[x(i, j)-y(i, j)]^{2}}
$$

\section{Neural Network Performance Evaluation}

The test set described in the previous section was used to evaluate KL-MOB. The classification outcome has four cases: True Positive (TP), False Positive (FP), True Negative (TN), and False Negative (FN). The metrics used to measure the performance are accuracy (ACC), sensitivity (TPR), specificity (SPC), and precision (PPV) and are defined as follows:

$$
\begin{aligned}
& \text { Accuracy }(A C C)=\frac{T P+T N}{T P+F P+T N+F N}, \\
& \text { Sensitivity }(T P R)=\frac{T P}{T P+F N},
\end{aligned}
$$




$$
\begin{aligned}
& \text { Specificity }(S P C)=\frac{T N}{F P+T N}, \\
& \text { Precision }(P P V)=\frac{T P}{T P+F P},
\end{aligned}
$$

The graph of true positive rate (TPR) and false positive rate (FPR) is the receiver operating characteristic (ROC) curve. The FPR is calculated as follows:

$$
\text { False Positive Rate }(F P R)=\frac{F P}{F P+T N} .
$$

\section{RESULTS}

In the experiments, noise reduction and contrast enhancement performance were evaluated independently, since they are two separate issues. The average value was computed for all images in each class. Tables 5 and 6 show the results for noise reduction and image enhancement, respectively. Figure 7 shows the noise reduction techniques that were applied to the original image and the hybrid method used in this work. Although the denoising filters could smooth and blur the resulting images, this can be enhanced by improving the image edges and by highlighting the high-frequency components to remove the residual noise. Figure 8 displays the original images and their enhanced versions.

\begin{tabular}{c|cc|cc|cc}
\multirow{2}{*}{ Method } & \multicolumn{2}{|c|}{ Covid19 } & \multicolumn{2}{c|}{ Normal } & \multicolumn{2}{c}{ Pneumonia } \\
\hline \multirow{3}{*}{ AMF } & PSNR & MAE & PSNR & MAE & PSNR & MAE \\
\cline { 2 - 7 } & 21.91 & 14.46 & 21.19 & 17.88 & 20.43 & 19.47 \\
NLMF & 20.47 & 19.19 & 20.41 & 19.41 & 20.40 & 19.40 \\
Proposed method & 22.04 & 14.38 & 21.21 & 17.59 & 20.45 & 19.32
\end{tabular}

Table 5. Average PSNR (db) and MAE for the various noise-reduction methods.

\begin{tabular}{c|cc|cc|cc} 
Method & \multicolumn{2}{|c|}{ Covid19 } & \multicolumn{2}{c|}{ Normal } & \multicolumn{2}{c}{ Pneumonia } \\
\hline \multirow{3}{*}{ CLAHE } & PSNR & MAE & PSNE & MAE & PSNR & MAE \\
\cline { 2 - 7 } Proposed method & 17.83 & 27.35 & 17.12 & 25.98 & 21.91 & 16.20 \\
& 19.14 & 23.13 & 17.28 & 25.45 & 22.11 & 16.01
\end{tabular}

Table 6. Average PSNR (db) and MAE for the various contrast-enhancement methods.

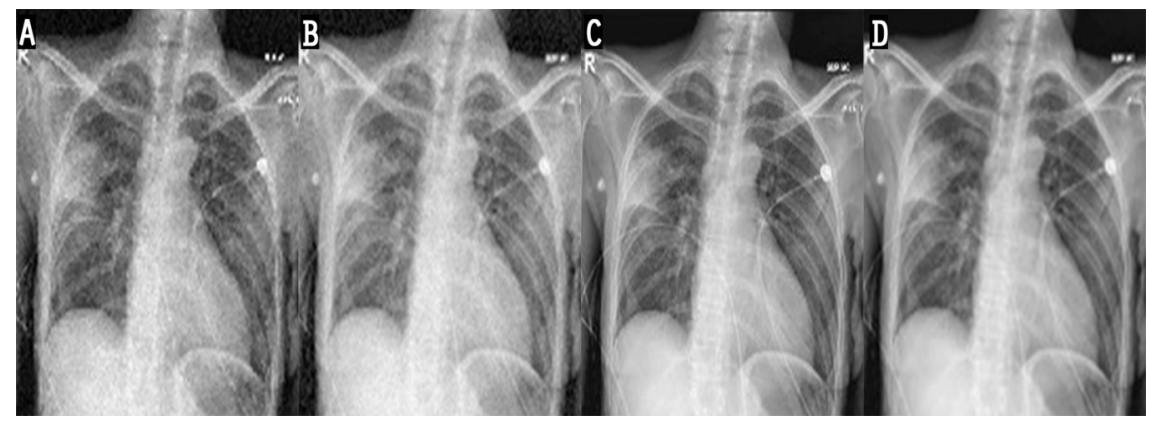

Figure 7. Result of noise-reduction techniques applied to images: (A) original image, (B) image denoised by AMF, (C) image denoised by NLMF, (D) image denoised by proposed method.

The performance of the proposed KL-MOB was evaluated separately for each class of the test set. Table 7 compares the performance of the KL-MOB model for the classification problem involving original 


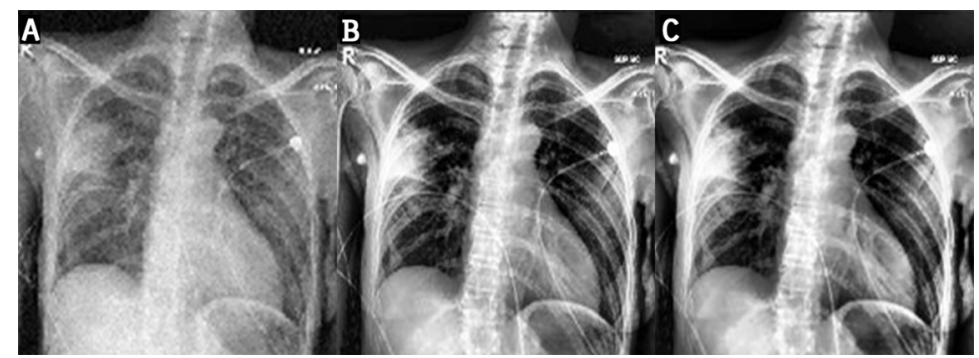

Figure 8. Results of image enhancement: (A) original image, (B) image enhanced by CLAHE, (C) image enhanced by proposed method.

\begin{tabular}{c|ccccc|ccccc} 
& \multicolumn{6}{|c|}{ Enahnced image } & \multicolumn{5}{c}{ Original image } \\
\cline { 2 - 11 } & ACC\% & PPV\% & SPC\% & TPR\% & MCC\% & ACC\% & PPV\% & SPC\% & TPR\% & MCC\% \\
\hline Covid19 & 99.87 & 99.00 & 99.93 & 99.00 & 98.93 & 92.61 & 96.83 & 99.13 & 74.39 & 80.60 \\
Normal & 98.24 & 98.30 & 97.85 & 98.64 & 96.53 & 97.11 & 98.17 & 98.99 & 93.86 & 93.77 \\
Pneumonia & 97.99 & 97.81 & 98.68 & 97.31 & 96.03 & 91.00 & 81.30 & 86.74 & 98.26 & 82.53 \\
Overall & 98.70 & 98.37 & 98.82 & 98.32 & 96.60 & 93.57 & 92.10 & 94.95 & 88.84 & 85.90
\end{tabular}

Table 7. Metrics for original images and for images enhanced by KL-MOB.

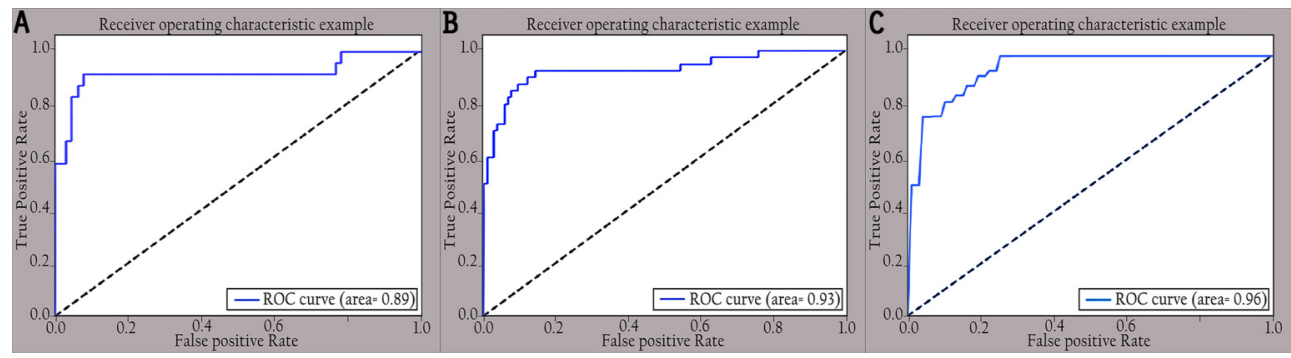

Figure 9. ROC curves of different classes for original images: (A) Covid-19, (B) normal, and (C) pneumonia.

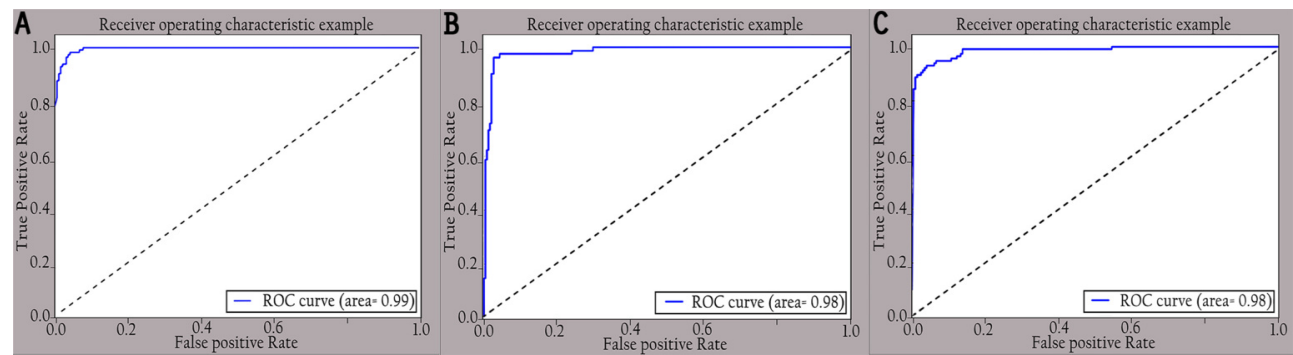

Figure 10. ROC curves of different classes for enhanced images: (A) Covid-19, (B) normal, and (C) pneumonia.

\section{DISCUSSION}

This work proposes an approach that combines noise-reduction algorithms with contrast enhancement. This approach introduces a type of hybrid filtering and contrast enhancement for the data set of images used for Covid-19 detection. The well-known measurable methods PSNR and MAE were used as image 
quality measurements for assessing and comparing image quality. The results of Table 5 show that using an AMF followed by a NLMF is entirely favorable for eliminating noise. The proposed hybrid algorithm is applied to the entire image instead of just parts of the image and preserves important details. Figure 11 illustrates the difference between the original CXRs and CXRs enhanced by applying the method proposed herein. Furthermore, we judge the lung damage in the enhanced image to be more perspicuous than in the original image. In addition, CLAHE with a bin of 256 gives the best PSNR, as shown in Table 6.

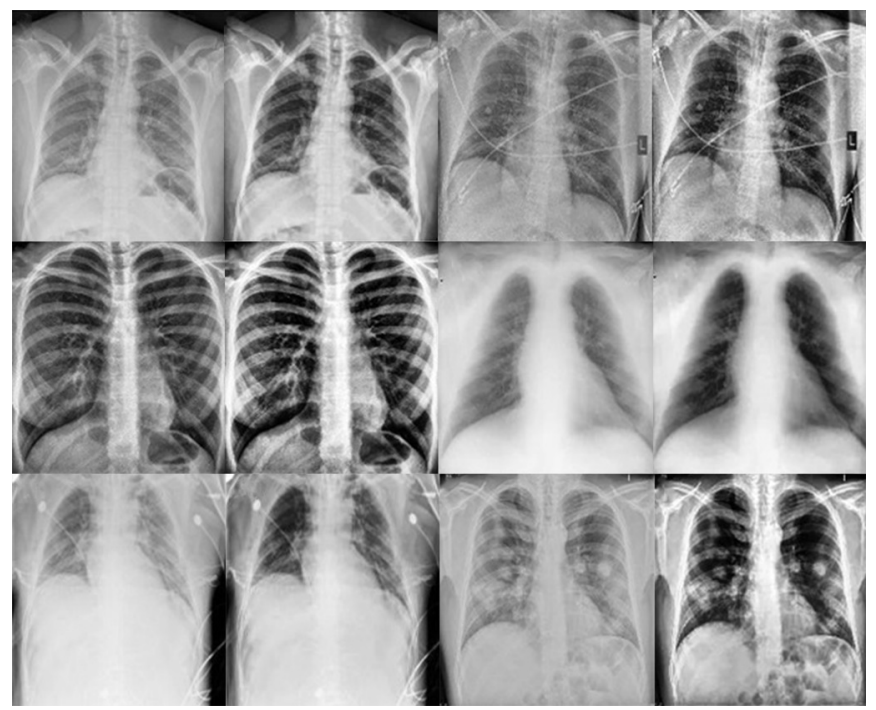

Figure 11. The first and third columns show the original images, and the second and fourth columns show the corresponding enhanced images.

To show the impact of the KL divergence loss on the efficacy of the proposed method, we performed several experiments using the categorical entropy loss function (CCE) and the mean square error (MSE) loss function. The results obtained in Table 8 show that the proposed method has a great impact on the performance of KL-MOB, thereby justifying the selection of the proposed network architecture and its associated training/learning schemes.

\begin{tabular}{c|c|cccc|cccc}
\multirow{2}{*}{ Model } & \multirow{2}{*}{ Loss function } & \multicolumn{4}{|c|}{ Enhanced image } & \multicolumn{4}{c}{ Original image } \\
\cline { 3 - 10 } & & ACC\% & PPV\% & SPC\% & TPR\% & ACC\% & PPV\% & SPC\% & TPR\% \\
\hline \multirow{3}{*}{ KL-MOB } & CCE & 96.79 & 95.22 & 97.60 & 95.42 & 90.14 & 87.94 & 92.23 & 83.05 \\
& MSE & 92.50 & 89.70 & 94.16 & 86.92 & 85.12 & 94.53 & 97.50 & 95.11 \\
& $\begin{array}{c}\text { Proposed } \\
\text { method }\end{array}$ & 98.70 & 98.37 & 98.82 & 98.32 & 93.57 & 92.10 & 94.95 & 88.84
\end{tabular}

Table 8. Performance on the test set with different loss functions.

Figure 12 shows the confusion matrix of the proposed network: all classes are identified with high true positives. Note that the Covid-19 cases are 99\% correctly classified by the KL-MOB model. Only $1 \%$ of Covid-19 cases are misclassified as pneumonia (non-Covid-19), and 1.4\% of the normal cases are misclassified as pneumonia. Only $0.2 \%$ of pneumonia (non-Covid-19) cases are wrongly classified as Covid-19. These results demonstrate that the proposed KL-MOB has a strong potential for detecting Covid-19. In particular, with limited Covid-19 cases, the results show that no confusion arises between normal patients and Covid-19 patients.

In our experiment of 100 patients with Covid-19, only one was misclassified with a 99.0\% PPV for Covid-19, which compares favorably with previous results of $98.9 \%$, and $96.12 \%$ for (Wang et al., 2020) and (Rezaul Karim et al., 2020), respectively. In addition, we compare the results obtained from the KL-MOB model with those from previous studies that used the same or similar datasets for evaluation (see Table 9). Not included in the comparison are studies that used smaller datasets (Farooq and Hafeez, 2020; Afshar et al., 2020; Hirano et al., 2020; Ucar and Korkmaz, 2020). The results show that, for all 


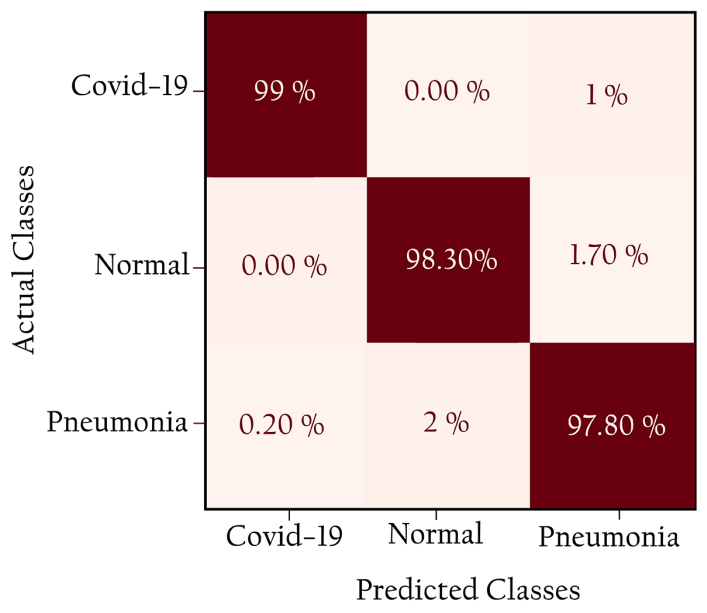

Figure 12. Confusion matrix for KL-MOB applied to COVIDx test dataset.

performance metrics [accuracy, sensitivity (TPR), specificity, and PPV for overall detection], the KLMOB model produces superior results compared with the models of (Wang et al., 2020) and (Rezaul Karim et al., 2020).

\begin{tabular}{c|c|cccc} 
Study & Classifier & ACC\% & SPC\% & TPR\% & PPV\% \\
\hline Wang et al. (2020) & COVID-Net (large) & 95.56 & 96.67 & 93.33 & 93.55 \\
Ahmed et al. (2020) & ReCoNet & 97.48 & 97.39 & 97.53 & 96.27 \\
Rezaul Karim et al. (2020) & DeepCOVIDExplainer & 98.11 & 98.19 & 95.06 & 96.84 \\
Proposed method & KL-MOB & 98.7 & 98.82 & 98.32 & 98.37 \\
\% Improvement & & 0.60 & 0.64 & 3.43 & 1.58
\end{tabular}

Table 9. Comparative performance of the various models with the improvement percentage compared to the state of art.

The promising deep learning models used for the detection of Covid-19 from radiography images indicate that deep learning likely still has untapped potential and can play a more significant role in fighting this pandemic. There is definitely still room for improvement through: (a) the other preprocesses such as increasing the number of images, implementing another preprocessing technique, i.e., data augmentation, utilizing different noise filters, and enhancement techniques. (b) design a model that deals with multiple inputs simultaneously, where utilizing multiple modalities may achieve superior outcomes than the individual modality (Zhang et al., 2021).

\section{CONCLUSION}

This work proposes a novel CNN-based MobileNet-structured neural network for detecting Covid-19 using COVIDx, which is the most widely used public dataset of CXR images to date. The evaluation of this approach shows that it outperforms the recent approach in terms of accuracy, specificity, sensitivity, and precision $(98.7 \%, 98 . \%, 98.32 \%$, and $98.37 \%$, respectively). The proposed method relies on image manipulation by applying a hybrid technique to enhance the visibility of CXR images. This advanced preprocessing technique facilitates the task of the KL-MOB model to extract features, allowing complex patterns in medical images to be recognized at a level comparable to that of an experienced radiologist. The KL divergence is used to boost the performance of the KL-MOB model, which outperforms recent approaches, as shown by the results. Where the KL divergence between the $\mu ; \sigma$ distribution and the prior is considered as a regularization, which aids to overcome the overfitting problem. Moreover, it is also believed that the notion of using KL divergence can be extended to other similar scenarios such as content-based image retrieval and fine-grained classification to improve the quality of object representation. Considering several essential factors such as the pattern by which Covid-19 infections spread, image acquisition time, scanner availability, and costs, we hope that these findings will make a useful contribution 
to the fight against Covid-19 and increase the acceptance of artificial-intelligence-assisted applications in clinical practice.

In future work, we will further enhance the proposed method's performance by including lateral views of CXR images in the training data because, in some cases, frontal-view CXR images do not permit a clear diagnosis of pneumonia cases. Besides, this work lacked in applying some of the techniques such as progressive resizing (Bhatt et al., 2021a), which can be applied to CNNs to carry out imaging-based diagnostics. Furthermore, visual ablation studies (Bhatt et al., 2021b; Joshi et al., 2021; Gite et al., 2021) can be performed along with deep learning, which will significantly improve the detection of Covid-19 manifestations in the CXR images. Since only a limited number of CXR images are available for Covid-19 infection, out-of-distribution issues may arise, so more data from related distributions is needed for further evaluation. There are several techniques that would be another way to overcome this problem, include, but are not limited to data augmentation techniques (Chaudhari et al., 2019), transfer learning (Taresh et al., 2021; Bhatt et al., 2021a), domain-adaptation (Zhang et al., 2020; Jin et al., 2021) and adversarial learning (Goel et al., 2021; Rahman et al., 2020; Motamed et al., 2021), etc. Finally, the image enhancement must be verified by a radiologist, which we have not yet been able to do due to the emerging conditions.

\section{ACKNOWLEDGMENTS}

This work was supported by the National Natural Science Foundation [61572177]. There was no additional external funding received for this study. 


\section{REFERENCES}

Afshar, P., Heidarian, S., Naderkhani, F., Oikonomou, A., Plataniotis, K. N., and Mohammadi, A. (2020). Covid-caps: A capsule network-based framework for identification of covid-19 cases from x-ray images. Pattern Recognition Letters, 138:638-643.

Ahmed, S., Yap, M. H., Tan, M., and Hasan, M. K. (2020). Reconet: Multi-level preprocessing of chest $\mathrm{x}$-rays for covid-19 detection using convolutional neural networks. medRxiv.

Albahli, S. (2020). A deep neural network to distinguish covid-19 from other chest diseases using x-ray images. Current Medical Imaging.

Alfasly, S. A. S., Hu, Y., Liang, T., Jin, X., Zhao, Q., and Liu, B. (2019). Variational representation learning for vehicle re-identification. In 2019 IEEE International Conference on Image Processing (ICIP), pages 3118-3122. IEEE.

Alghaili, M., Li, Z., and Ali, H. A. (2020). Facefilter: Face identification with deep learning and filter algorithm. Scientific Programming, 2020.

Apostolopoulos, I. D., Aznaouridis, S. I., and Tzani, M. A. (2020). Extracting possibly representative covid-19 biomarkers from x-ray images with deep learning approach and image data related to pulmonary diseases. Journal of Medical and Biological Engineering, 40:462-469.

Apostolopoulos, I. D. and Mpesiana, T. A. (2020). Covid-19: automatic detection from x-ray images utilizing transfer learning with convolutional neural networks. Physical and Engineering Sciences in Medicine, 43(2):635-640.

Bhatt, A., Ganatra, A., and Kotecha, K. (2021a). Covid-19 pulmonary consolidations detection in chest $\mathrm{x}$-ray using progressive resizing and transfer learning techniques. Heliyon, page e 07211.

Bhatt, A. R., Ganatra, A., and Kotecha, K. (2021b). Cervical cancer detection in pap smear whole slide images using convnet with transfer learning and progressive resizing. PeerJ Computer Science, 7:e348.

Canayaz, M. (2021). Mh-covidnet: Diagnosis of covid-19 using deep neural networks and meta-heuristicbased feature selection on x-ray images. Biomedical Signal Processing and Control, 64:102257.

Chaudhari, P., Agrawal, H., and Kotecha, K. (2019). Data augmentation using mg-gan for improved cancer classification on gene expression data. Soft Computing, pages 1-11.

Chowdhury, M. E. H., Rahman, T., Khandakar, A., Mazhar, R., Kadir, M. A., Mahbub, Z. B., Islam, K. R., Khan, M. S., Iqbal, A., Emadi, N. A., Reaz, M. B. I., and Islam, M. T. (2020). Can ai help in screening viral and covid-19 pneumonia? IEEE Access, 8:132665-132676.

Civit-Masot, J., Luna-Perejón, F., Domínguez Morales, M., and Civit, A. (2020). Deep learning system for covid-19 diagnosis aid using X-ray pulmonary images. Applied Sciences, 10(13):4640.

El Asnaoui, K. and Chawki, Y. (2020). Using X-ray images and deep learning for automated detection of coronavirus disease. Journal of Biomolecular Structure and Dynamics, pages 1-12.

El-bana, S., Al-Kabbany, A., and Sharkas, M. (2020). A multi-task pipeline with specialized streams for classification and segmentation of infection manifestations in covid-19 scans. PeerJ Computer Science, 6:e303.

Farooq, M. and Hafeez, A. (2020). Covid-resnet: A deep learning framework for screening of covid19 from radiographs. arXiv preprint arXiv:2003.14395.

Feng, L., Wang, H., Jin, B., Li, H., Xue, M., and Wang, L. (2018). Learning a distance metric by balancing kl-divergence for imbalanced datasets. IEEE Transactions on Systems, Man, and Cybernetics: Systems, 49(12):2384-2395.

Gite, S., Khatavkar, H., Kotecha, K., Srivastava, S., Maheshwari, P., and Pandey, N. (2021). Explainable stock prices prediction from financial news articles using sentiment analysis. PeerJ Computer Science, 7:e340.

Goel, T., Murugan, R., Mirjalili, S., and Chakrabartty, D. K. (2021). Automatic screening of covid-19 using an optimized generative adversarial network. Cognitive computation, pages 1-16.

Haghanifar, A., Majdabadi, M. M., Choi, Y., Deivalakshmi, S., and Ko, S. (2020). Covid-cxnet: Detecting covid-19 in frontal chest $\mathrm{x}$-ray images using deep learning. arXiv preprint arXiv:2006.13807.

Hemdan, E. E.-D., Shouman, M. A., and Karar, M. E. (2020). Covidx-net: A framework of deep learning classifiers to diagnose covid-19 in x-ray images. arXiv preprint arXiv:2003.11055.

Hirano, H., Koga, K., and Takemoto, K. (2020). Vulnerability of deep neural networks for detecting covid-19 cases from chest X-ray images to universal adversarial attacks. Plos one, 15(12): 0243963.

Horry, M. J., Chakraborty, S., Paul, M., Ulhaq, A., Pradhan, B., Saha, M., and Shukla, N. (2020). Covid-19 detection through transfer learning using multimodal imaging data. IEEE Access, 8:149808-149824. 
Howard, A. G., Zhu, M., Chen, B., Kalenichenko, D., Wang, W., Weyand, T., Andreetto, M., and Adam, H. (2017). Mobilenets: Efficient convolutional neural networks for mobile vision applications. arXiv preprint arXiv:1704.04861.

Huang, R.-Y., Dung, L.-R., Chu, C.-F., and Wu, Y.-Y. (2016). Noise removal and contrast enhancement for X-ray images. Journal of Biomedical Engineering and Medical Imaging, 3(1):56.

Ikhsan, I. A. M., Hussain, A., Zulkifley, M. A., Tahir, N. M., and Mustapha, A. (2014). An analysis of $\mathrm{x}$-ray image enhancement methods for vertebral bone segmentation. In 2014 IEEE 10th International Colloquium on Signal Processing and its Applications, pages 208-211. IEEE.

Jin, Q., Cui, H., Sun, C., Meng, Z., Wei, L., and Su, R. (2021). Domain adaptation based self-correction model for covid-19 infection segmentation in ct images. Expert Systems with Applications, 176:114848.

Joshi, G., Walambe, R., and Kotecha, K. (2021). A review on explainability in multimodal deep neural nets. IEEE Access.

Khare, V. and Chugh, S. (2014). An efficient adaptive median filtering approach for the removal of impulse noise. In 2014 International Conference on Advances in Engineering \& Technology Research (ICAETR-2014), pages 1-5. IEEE.

Kim, K., Choi, J., and Lee, Y. (2020). Effectiveness of non-local means algorithm with an industrial 3 mev linac high-energy x-ray system for non-destructive testing. Sensors, 20(9):2634.

Kingma, D. P. and Ba, J. (2014). Adam: A method for stochastic optimization. arXiv preprint arXiv:1412.6980.

Kingma, D. P. and Welling, M. (2013). Auto-encoding variational bayes. arXiv preprint arXiv:1312.6114.

Luz, E., Silva, P. L., Silva, R., Silva, L., Moreira, G., and Menotti, D. (2020). Towards an effective and efficient deep learning model for covid-19 patterns detection in x-ray images. arXiv preprint arXiv:2004.05717.

Lv, D., Wang, Y., Wang, S., Zhang, Q., Qi, W., Li, Y., and Sun, L. (2021). A cascade-seme network for covid-19 detection in chest X-ray images. Medical Physics, 48(5):2337-2353.

Maghdid, H. S., Asaad, A. T., Ghafoor, K. Z., Sadiq, A. S., and Khan, M. K. (2020). Diagnosing covid-19 pneumonia from $\mathrm{x}$-ray and ct images using deep learning and transfer learning algorithms. arXiv preprint arXiv:2004.00038.

Maguolo, G. and Nanni, L. (2021). A critic evaluation of methods for covid-19 automatic detection from x-ray images. Information Fusion, 76:1-7.

Medhi, K., Jamil, M., and Hussain, M. I. (2020). Automatic detection of covid-19 infection from chest $\mathrm{x}$-ray using deep learning. medRxiv.

Mirzabagheri, M. (2017). A new adaptive method for removing impulse noise from medical images. Signal Processing and Renewable Energy, 1(1):37-45.

Motamed, S., Rogalla, P., and Khalvati, F. (2021). Randgan: randomized generative adversarial network for detection of covid-19 in chest x-ray. Scientific Reports, 11(1):1-10.

Narin, A., Kaya, C., and Pamuk, Z. (2020). Automatic detection of coronavirus disease (covid-19) using $\mathrm{x}$-ray images and deep convolutional neural networks. arXiv preprint arXiv:2003.10849.

Ning, C.-Y., Liu, S.-f., and Qu, M. (2009). Research on removing noise in medical image based on median filter method. In 2009 IEEE International Symposium on IT in Medicine \& Education, volume 1, pages 384-388. IEEE.

Ozturk, T., Talo, M., Yildirim, E. A., Baloglu, U. B., Yildirim, O., and Acharya, U. R. (2020). Automated detection of covid-19 cases using deep neural networks with x-ray images. Computers in Biology and Medicine, page 103792.

Paul, E. M., Perumal, B., and Rajasekaran, M. P. (2018). Filters used in x-ray chest images for initial stage tuberculosis detection. In 2018 International Conference on Inventive Research in Computing Applications (ICIRCA), pages 235-239. IEEE.

Punn, N. S. and Agarwal, S. (2021). Automated diagnosis of covid-19 with limited posteroanterior chest X-ray images using fine-tuned deep neural networks. Applied Intelligence, 51(5):2689-2702.

Qi, X., Brown, L. G., Foran, D. J., Nosher, J., and Hacihaliloglu, I. (2021). Chest X-ray image phase features for improved diagnosis of covid-19 using convolutional neural network. International journal of computer assisted radiology and surgery, 16(2):197-206.

Rabbouch, H., Messaoud, O. B., and Saâdaoui, F. (2020). Multi-scaled non-local means parallel filters for medical image denoising. In International Conference on Algorithms and Architectures for Parallel Processing, pages 606-613. Springer. 
Rahman, A., Hossain, M. S., Alrajeh, N. A., and Alsolami, F. (2020). Adversarial examples-security threats to covid-19 deep learning systems in medical iot devices. IEEE Internet of Things Journal.

Rahman, T., Khandakar, A., Qiblawey, Y., Tahir, A., Kiranyaz, S., Abul Kashem, S. B., Islam, M. T., Al Maadeed, S., Zughaier, S. M., Khan, M. S., and Chowdhury, M. E. (2021). Exploring the effect of image enhancement techniques on covid-19 detection using chest x-ray images. Computers in Biology and Medicine, 132:104319.

Raj, V. P. and Venkateswarlu, T. (2012). Denoising of magnetic resonance and x-ray images using variance stabilization and patch based algorithms. The International Journal of Multimedia \& Its Applications, 4(6):53.

Ramadhan, M. M., Faza, A., Lubis, L. E., Yunus, R. E., Salamah, T., Handayani, D., Lestariningsih, I., Resa, A., Alam, C. R., Prajitno, P., Pawiro, S. A., Sidipratomo, P., and Soejoko, D. S. (2020). Fast and accurate detection of covid-19-related pneumonia from chest X-ray images with novel deep learning model. arXiv preprint arXiv:2005.04562.

Rezaul Karim, M., Döhmen, T., Rebholz-Schuhmann, D., Decker, S., Cochez, M., and Beyan, O. (2020). Deepcovidexplainer: Explainable covid-19 diagnosis based on chest $\mathrm{x}$-ray images. arXiv e-prints, pages arXiv-2004.

Saiz, F. A. and Barandiaran, I. (2020). Covid-19 detection in chest X-ray images using a deep learning approach. International Journal of Interactive Multimedia and Artificial Intelligence, InPress (InPress), 1.

Sawant, A., Zeman, H., Muratore, D., Samant, S., and DiBianca, F. (1999). Adaptive median filter algorithm to remove impulse noise in $x$-ray and CT images and speckle in ultrasound images, volume 3661, pages 1263-1274. Society of Photo-Optical Instrumentation Engineers, ii edition. Proceedings of the 1999 Medical Imaging - Image Processing ; Conference date: 22-02-1999 Through 25-02-1999.

Senthilkumar, R. and Senthilmurugan, M. (2014). Triad histogram to enhance chest x-ray image. Int $J$ Adv Res Comput Commun Eng, 3:8577-80.

Sethy, P. K. and Behera, S. K. (2020). Detection of coronavirus disease (covid-19) based on deep features. Preprints, 2020030300:2020.

Siddhartha, M. and Santra, A. (2020). Covidlite: A depth-wise separable deep neural network with white balance and clahe for detection of covid-19. arXiv preprint arXiv:2006.13873.

Sonali, Sahu, S., Singh, A. K., Ghrera, S., and Elhoseny, M. (2019). An approach for de-noising and contrast enhancement of retinal fundus image using clahe. Optics \& Laser Technology, 110:87-98.

Su, C., Ju, S., Liu, Y., and Yu, Z. (2015). Improving part algorithm with kl divergence for imbalanced classification. Intelligent Data Analysis, 19(5):1035-1048.

Taresh, M. M., Zhu, N., Ali, T. A. A., Hameed, A. S., and Mutar, M. L. (2021). Transfer learning to detect covid-19 automatically from x-ray images using convolutional neural networks. International Journal of Biomedical Imaging, 2021.

Tartaglione, E., Barbano, C. A., Berzovini, C., Calandri, M., and Grangetto, M. (2020). Unveiling covid-19 from chest x-ray with deep learning: a hurdles race with small data. International Journal of Environmental Research and Public Health, 17(18):6933.

Ucar, F. and Korkmaz, D. (2020). Covidiagnosis-net: Deep bayes-squeezenet based diagnosis of the coronavirus disease 2019 (covid-19) from x-ray images. Medical Hypotheses, 140:109761.

Wang, L., Lin, Z. Q., and Wong, A. (2020). Covid-net: a tailored deep convolutional neural network design for detection of covid-19 cases from chest X-ray images. Scientific Reports, 10(1):19549.

Wang, S.-H., Zhang, Y., Cheng, X., Zhang, X., and Zhang, Y.-D. (2021). Psspnn: Patchshuffle stochastic pooling neural network for an explainable diagnosis of covid-19 with multiple-way data augmentation. Computational and Mathematical Methods in Medicine, 2021.

Wen, H., Qi, W., and Shuang, L. (2016). Medical x-ray image enhancement based on wavelet domain homomorphic filtering and clahe. In 2016 International Conference on Robots \& Intelligent System (ICRIS), pages 249-254. IEEE.

Yu, D., Xu, Q., Guo, H., Zhao, C., Lin, Y., and Li, D. (2020). An efficient and lightweight convolutional neural network for remote sensing image scene classification. Sensors, 20(7):1999.

Zhang, Y., Niu, S., Qiu, Z., Wei, Y., Zhao, P., Yao, J., Huang, J., Wu, Q., and Tan, M. (2020). Covid-da: Deep domain adaptation from typical pneumonia to covid-19. arXiv preprint arXiv:2005.01577.

Zhang, Y.-D., Zhang, Z., Zhang, X., and Wang, S.-H. (2021). Midcan: A multiple input deep convolutional attention network for covid-19 diagnosis based on chest ct and chest X-ray. Pattern Recognition Letters. 
Zhou, X., Zheng, Y., Tan, L., and Zhao, J. (2016). Medical image contrast enhancement via wavelet homomorphic filtering transform. TELKOMNIKA (Telecommun. Comput. Electron. Control.), 14:1203. 